of the showing factors loadings higher than 0.6: "conditions related to chemical exposure during pregnancy", which explained $20 \%$ of the variance; "lifestyle exposures", such as smoking and hair dyes and hair cosmetics use during pregnancy, explaining $17 \%$ of the total variance; and "consumption of health services during pregnancy", such as x-rays and delivery type (cesarean or vaginal delivery), explaining $15 \%$ of the total variance. Logistic modelling revealed statistically significant association between childhood leukaemia and chemical exposure during pregnancy $(\mathrm{OR}=1.36$; $95 \%$ CI 1.16 to 1.59), and also with consumption of health services during pregnancy $(\mathrm{OR}=1.27 ; 95 \% \mathrm{CI} 1.08$ to 1.49$)$. The observed results are suggestive of the contribution of environmental exposures to childhood leukaemia development, not just individually, which has been supported by the literature according to carcinogenesis in general, and to leukaemogenesis in particular, as resulting from several mutations triggered by joint environmental exposures.

\section{P2-84 CHRONIC CONDITIONS AND MAJOR DEPRESSION IN COMMUNITY-DWELLING OLDER ADULTS}

doi:10.1136/jech.2011.142976i.19

${ }^{1} \mathrm{~K}$ Fiest, ${ }^{* 1,2} \mathrm{~S}$ Currie, ${ }^{1} \mathrm{~J}$ Williams, ${ }^{1} \mathrm{~J}$ Wang. ${ }^{1}$ University of Calgary, Calgary, Alberta, Canada; ${ }^{2}$ Alberta Health Services, Calgary, Alberta, Canada

Objectives To estimate (1) the prevalence of long-term medical conditions and of comorbid major depression, and (2) the associations between major depression and various chronic medical conditions in a general population of older adults (over 50 years of age) and in persons who are traditionally classified as seniors (65 years and older).

Methods Data from the Canadian Community Health SurveyMental Health and Wellbeing (CCHS-1.2) were analysed. For the purposes of these analyses the dataset was restricted to those aged 50 and over $(n=15591)$. Chronic health conditions were assessed using a self-report method of doctor diagnosis. The World Mental Health-Composite Diagnostic Interview was used to assess major depressive episodes based on DSM-IV criteria.

Results The overall prevalence of having at least one chronic condition in those over 50 years of age was $82.4 \%$, compared to $62.0 \%$ in those under 50 . The prevalence of a major depressive episode in those over 50 with one chronic condition was $3.7 \%$, compared with $1.0 \%$ in those without a long-term medical condition. The top 3 chronic health conditions in seniors aged 65 or older were arthritis/rheumatism, high blood pressure and back problems. Chronic Fatigue Syndrome, fibromyalgia and migraine headache had the highest comorbidity with major depression in the senior population.

Conclusions Differences were found between rates of chronic conditions and major depression between the general population, older adults and seniors in this study. Primary and secondary prevention efforts should target seniors who exhibit symptoms of depression or highly prevalent chronic health conditions.

\section{P2-85 DIABETES SELF-CARE: “NO WAY TO DO EVERYTHING"}

doi:10.1136/jech.2011.142976i.20

${ }^{1} \mathrm{R}$ Figueiredo, ${ }^{*} \mathrm{~A}$ Hernáez, ${ }^{1} \mathrm{M}$ de Fátima Diniz, ${ }^{1} \mathrm{~S}$ Barreto. ${ }^{1} \mathrm{UFMG}$, Belo Horizonte, Minas Gerais, Brazil; ${ }^{2}$ Universidad Rovira i Virgili, Barcelona, Catalunya, Spain

Introduction Individuals' opinions, believes and experiences with diabetes influence how they implement the regime prescribed for the disease in their daily lives. Very often, the prescribed regime imposes changes and disciplines that are not feasible or do not fit to individual's routines and possibilities, compromising their adherence to self-care behaviours and diabetes control.

Objectives To uncover the explanatory models of diabetes built by patients and to understand how these models relate to their attitudes and thoughts regarding their diabetes regimes.

Methods We conducted two focus groups with patients with diabetes, both Type 1 and Type 2, in two different health services in the city of Belo Horizonte - Minas Gerais. The number of participants ranged from 4 to 7 , two females and five males. The analytical strategy is rooted in the Grounded Theory. Atlas-ti software, version 6,was employed.

Results Five themes emerged from the analysis regarding participants' perceptions: "Diabetes: shock, anger, sadness," "emotional state and diabetes: cause and effect", "managing diabetes: a sacrifice", "diet: the greatest dilemma" and "insulin: ignorance and fear". It is possible to grasp a bidirectional relationship between emotional disorders and diabetes. Compliance with the prescribed diet is the most challenging aspect of diabetes care and we see great dissatisfaction with the use of insulin, mainly by associating its use with the worsening of the disease and with hypoglycemia.

Conclusion We noticed that participants struggle to integrate selfcare activities in their everyday life. How they react to this integration process varies according to their psychological barriers.

\section{P2-86 EDUCATION AND SURVIVAL OF NON-HODGKIN LYMPHOMA IN DENMARK}

doi:10.1136/jech.2011.142976i.21

${ }^{1} \mathrm{~B}$ L Frederiksen, ${ }^{*} \mathrm{P}$ de Nully Brown, ${ }^{3} \mathrm{~S} 0$ Dalton, ${ }^{3} \mathrm{M}$ Stedding-Jessen, ${ }^{1} \mathrm{M}$ Osler. ${ }^{1}$ Research Centre for Prevention and Health, Glostrup University Hospital, Capital Region of Copenhagen, Glostrup, Denmark; ${ }^{2}$ Department of Haematology, Copenhagen University Hospital, Copenhagen, Denmark; Institute of Cancer Epidemiology, Danish Cancer Society, Copenhagen, Denmark

Introduction This study examined socio-economic inequalities in survival after non-Hodgkin lymphoma, and to what extent any disparities were explained by differences in comorbidity, disease severity at the time of diagnosis, and the treatment given.

Methods Registry-based nationwide cohort study based on 5738 persons diagnosed 2000-2008 from the Danish national lymphoma database and linked for individual socioeconomic information in Statistics Denmark.

Results Long-term mortality was highest in patients with a short education as compared to those higher educated. The social difference among patient $\leq 80$ years was increasing over time ( $p_{\text {interaction }}=0.01$ ). Thus, in the period 2000-2004 the HR among the short educated compared with the higher educated was 1.47 (95\% CI 1.34 to 1.61$)$ and increased to $H R=1.70$ (95\% CI 1.37 to 2.11$)$ in 2005-2008. However, no educational differences were seen among the oldest patients of 81 years and above. The educational gradient was attenuated by including comorbidity in the models, and further slightly attenuated by including lymphoma-specific prognostic factors (stadium, elevated lactatdehydrogenase, extranodal involvement). However, the educational gradient was still significant, as was the interaction with calendar period $\left(p_{\text {interaction }}=0.03\right)$. No socio-economic differences were found with respect to treatment with chemotherapy, radiation, or immunotherapy.

Conclusion Differences in survival among NHL patients with different socio-economic position are partly caused by differences in comorbidity and the severity of disease at the time of diagnosis, while no differences in treatment were found. The increasing social gradient over time may be partly explained by a more pronounced decrease in lifestyle-associated comorbidity among higher social groups in recent years. 